Case Report

\title{
FIBROMA OF OVARY PRESENTING AS M EIGS' SYNDROME - A CASE REPORT
}

\author{
Aparna Rajesh ${ }^{1}$, Deepa Kanagal ${ }^{2}$, Harish Shetty ${ }^{3}$, Prasanna Shetty ${ }^{4}$ \\ ${ }^{1}$ Assistant Professor, ${ }^{2}$ Associate Professor, ${ }^{3}$ Prof and Head, ${ }^{4}$ Professor \\ Correspondence: \\ Aparna Rajesh, \\ Assistant Professor, Department of OBG, \\ K.S. Hegde M edical Academy, Nitte University, Mangalore - 575018 \\ M obile : +9194482 35280, E-M ail: drpannarajesh@gmail.com
}

Department of Obstetrics and gynecology, K.S. Hegde M edical Academy, Nitte University, Karnataka, India - 575018.

\begin{abstract}
:
M eigs' syndrome is a rare but well known syndrome defined as the association of ascites, pleural effusion and a benign solid ovarian tumor usually a fibroma in which tumor removal leads to complete resolution of pleural and peritoneal effusions. We report a case of M eigs' syndrome in a post menopausal woman which mimicked ovarian malignancy creating a diagnostic dilemma where the lady had complete recovery after surgery. Considering the good prognosis of M eigs' syndrome, prompt and accurate diagnosis is necessary to differentiate the syndrome from other ovarian malignancies.
\end{abstract}

Keywords: M eigs' syndrome, ascites, pleural effusion, ovarian fibroma

\section{Introduction:}

Meigs's syndrome is the triad of ovarian fibroma with ascites and pleural effusion. It is a rare but well known syndrome. Pleural effusion and ascites resolve with successful resection of the ovarian tumor. Although M eig's syndrome mimics a malignant condition, it is a benign disease and has a very good prognosis if properly managed. Life expectancy after surgical removal of the tumor mirrors that of the general population.

\section{Case Report :}

A 53 year old postmenopausal woman presented to us with abdominal distention and progressively increasing breathlessness since 1 month. She had attained menopause 12 years back. There were no post menopausal symptoms. Clinical examination revealed a mass of 28 week's gravid uterine size occupying the lower abdomen

\begin{tabular}{|c|}
\hline Access this article online \\
\hline Quick Response Code \\
\hline
\end{tabular}
extending above the umbilicus. The mass was firm to hard in consistency with restricted mobility. There was associated ascites. Pelvic examination showed the mass to be separate from the uterus.
Examination of the respiratory system revealed decreased air entry on the right side.

Ultrasonography revealed a large solid, hypo echoic mass lesion in the lower abdomen measuring $12 \times 12 \mathrm{cms}$ arising from the ovary associated with ascites and right pleural effusion. Chest radiography confirmed pleural effusion. (Fig.1) Pleural fluid and ascitic fluid cytology showed the presence of reactive mesothelial cells with no malignant cells. The level of tumor marker CA 125 was in the normal range. The lady was taken for exploratory laparotomy in view of the ovarian tumor.

On laparotomy there was a solid left ovarian tumor of $13 x$ $12 \mathrm{cms}$ with ascites. (Fig. 2) The right ovary and uterus were normal. About 2 liters of ascitic fluid was drained. Total abdominal hysterectomy with bilateral salpingo oophorectomy was done and the specimen was sent for histopathology. (Fig. 3, 4)The other abdominal organs were found to be normal. Histopathology confirmed the diagnosis of ovarian fibroma and the absence of malignant cells. (Fig.5)The post operative period of the patient was uneventful. The pleural effusion regressed after surgery. The lady was discharged on the eighth post operative day. She is on regular follow up and is asymptomatic. 


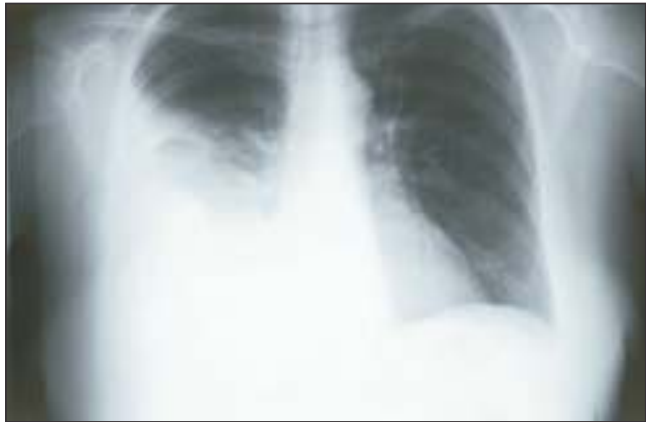

Fig 1: Chest X-ray showing right pleural effusion

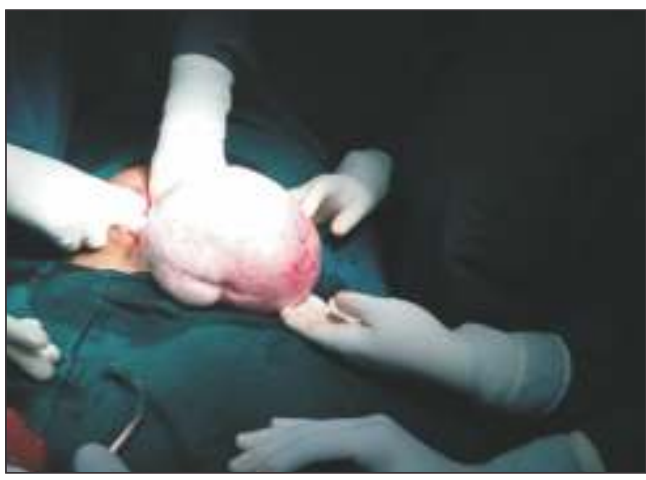

Fig. 2: Ovarian Fibroma seen during surgery

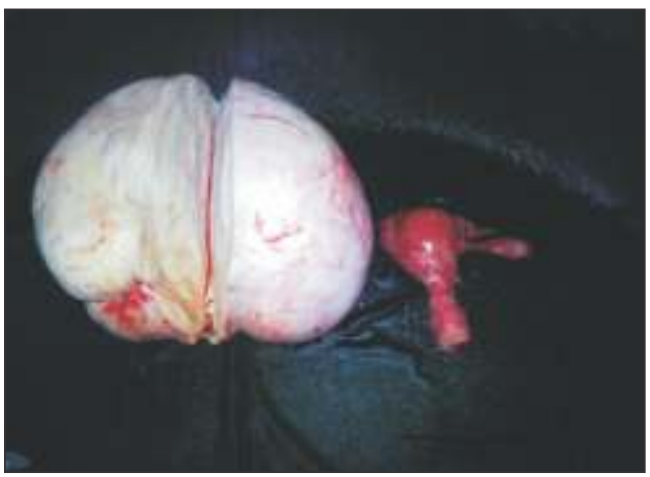

Fig. 3: Ovarian Fibroma specimen

\section{Discussion :}

Salmon in 1934 described the association of pleural effusion with benign pelvic tumors. In 1937, Meigs' and Cass described 7 cases of ovarian fibromas associated with ascites and pleural effusion. (1) This syndrome has been named after M eigs' and must fulfill the minimal criteria of pleural effusion, solid ovarian tumor and clearing of effusion after removal of the tumor.

Fibromas account for $4 \%$ of ovarian neoplasms .They present during the fifth and sixth decade. 10 to $15 \%$ of all fibromas are associated with ascites while only $1 \%$ have

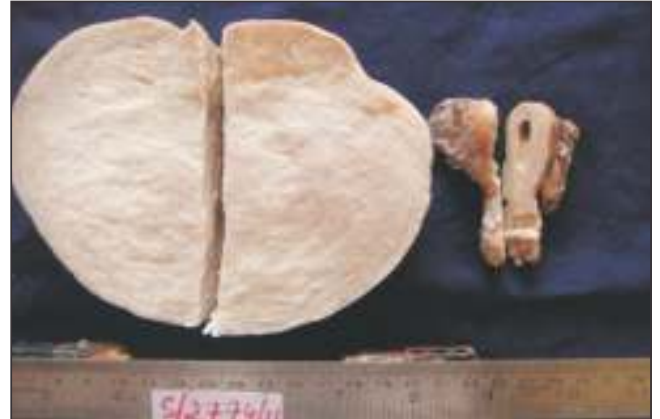

Fig. 4: Cut Section

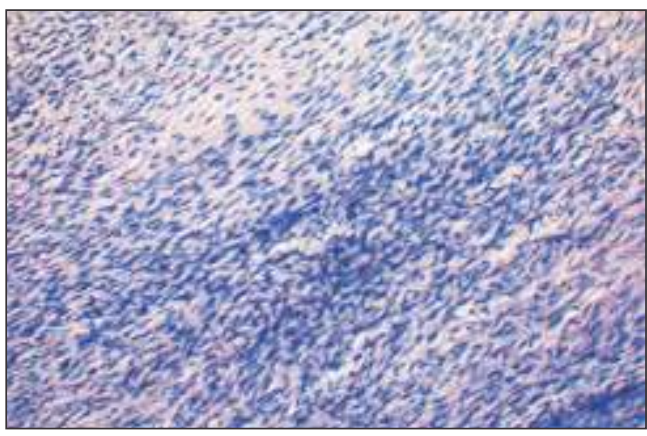

Fig.5: Histopathology

pleural effusion in addition to ascites.(2) These tumors have an extremely low malignant potential . Other pelvic tumors such as Brenner tumor and Granulosa cell tumor can be associated with ascites and pleural effusion and is described as pseudo meig's syndrome. $(3,4)$

Meigs suggested that the fluid in the abdomen originated from the edematous fibroma that can leak fluid (1). A discrepancy between the arterial supply to large mass of tumor tissue and its limited venous and lymphatic drainage may contribute to stromal edema and transudation (5). The ascitic fluid collection, related to benign ovarian tumor is thought to be caused by excessive transudate from the tumor surface in a degree that the peritoneum cannot absorb (3).

Diagnosis is essentially clinical. Cytomorphology of the fluids and Serum Ca125 levels may be used to rule out malignant nature of the lesion. The work up should include ultrasound of the pelvis, CT of the chest, abdomen and pelvis, M RI of the pelvis, sampling of the pleural as well as the ascitic fluid and serum markers of malignancy like Ca125 $(3,6)$. 
Surgery is curative and life expectancy is similar to the general population. In our patient there was complete resolution of ascites and pleural effusion after surgery.

\section{References:}

1. M eigs JV, Cass JW, "Fibroma of the ovary with ascites and hydrothorax with report of seven cases".Am J Obstet Gynecol 1937, 33: 249-266.

2. Lanitis S, Sivakumar S, Behranwala K, Zacharakis E, Mufti R.A, Hadjiminas D.J. "A case of Meigs syndrome mimicking metastatic breast carcinoma" World J Surgical Oncol 2009,7:10.

3. Abad A, Cazorla E, Ruiz F, Aznar I, Asins E, Llixiona J. “M eigs' syndrome with elevated CA 125: case report and review of literature" Eur J Obstet \& Gynecol reprod Biol" 1999, 82: 97-99.

4. Fuji M, Okino M, Fujioka K, Yamashita K, Hamano K. "Psuedo M eigs' syndrome caused by breast cancer metastasis to both ovaries" Breast cancer 2006, 13: 344-348.

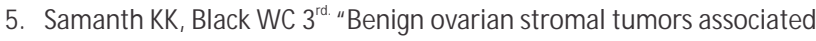
with free peritoneal fluid" Am J Obstet Gynecol 1970; 107: 538-45.

6. Nemeth AJ, Patel SK. "M eigs syndrome revisited" J Thorac Imaging 2003; 18:100-103. 